\title{
Research on the Methods to Improve the Computer Network Reliability Xinyu Zheng ${ }^{1, a}$ Zhengliang Liu $^{1, a}$ \\ ${ }^{12}$ Gannan Medical University, Ganzhou, Jiangxi, China, 341000
}

\author{
Keywords: Methods, Improvement, Computer Network, Reliability
}

\begin{abstract}
With the advent of the information age, the era of computer network has penetrated people's daily lives, in people's daily life and work is playing an increasingly large role in the development of various social sectors in the field of computer applications are also inseparable. With the security and reliability issues from the computer network has become the key to affect the computer network development. This article on how to improve the reliability of methods to make computer networks discussed.
\end{abstract}

\section{Introduction}

In recent years, with the acceleration continues to be put into use and computer networking process of computer products, the use of computer network users increases every year, though that computer network system has formed a relatively complete and sound system, but in the process of network constantly changing, along with the network of safety and reliability requirements are also increasing reliability of computer networks much attention, because the reliability of the computer network is a measure of the overall performance of modern computer network technology indicators. So, in terms of the status quo, improve the reliability of the computer network, it is necessary, has practical significance.

In order to ensure the use of computer networks from outside interference, to ensure the smooth running of the environment in the state, we need to ensure the reliability of the computer network, which has become a matter of growing concern. Computer network system to measure the quality of the merits of the indicators is the network reliability. In the design and research of computer network technology, the reliability is the most important issue, only the reliability of the computer network system of research and design, computer networks will be able to run in a stable state. Thus, the reliability is very important theoretical and practical significance to improve the computer network.

\section{The Meaning of Computer Network Reliability}

Reliability refers to a computer network in a limited time and conditions, several different parts of the computer connected via a communication device, in the role of network operation and related network management software, to complete the assigned time and under defined conditions function to accomplish the transfer and sharing of information between the resources to achieve the operation of the network. On the whole, the different parts of a computer network are multiple computers and set a good external communication device connection into a powerful communication system, so that the realization of resource sharing between multiple computers, software, hardware, data and information sharing. Improve the reliability of the computer network for the stable development of the whole country and society, as well as economic and property safety is of great significance, but also to protect the personal safety of users of computer networks important prerequisite.

\section{The Meaning of Exploring Computer Network Reliability}

In today's rapid development of information technology, security and reliability of computer network computer network is a prerequisite for survival. We use a computer network to transmit information to the final client, which has brought enormous convenience for people's life and work, 
so that people get the resources and information sharing. However, as long as the existence of a computer network, you cannot prevent the invasion of hackers, viruses, harm to the safety and reliability of the network, resulting in the omission of information that people, property and even endangering national economic security. Therefore, improving the reliability of the network to enhance network security, hacker resist viruses become clean network environment a top priority, so that customers can feel at ease, convenient to use the Internet computer network, which is the study of computer networks to achieve the desired reliability.

\section{The Principles of Computer Network Reliability}

To improve the reliability of the computer network, it is necessary to grasp the basic principles of network control principles and design, and design in accordance with the relevant technical specifications, in order to achieve to improve network reliability. The author believes that to achieve the reliability of computer network design should follow the following principles:

Utilizes the Principle of Redundant Design. Network design, in order to ensure information security system, you must choose better performance, higher safety factor, greater reliability of computer servers and network processing products. In the selection process, try to choose a powerful redundant fault-tolerant features a total of computer network products, which can effectively avoid the problem of computer network systems because the network failure.

The Open Network Architecture. In the design process of the network, according to the technical standards for the use of open computer network architecture, because this architecture supports heterogeneous systems and interoperability of heterogeneous devices, can effectively improve the ability to expand and upgrade the network.

Have Advanced Design Consciousness. In the design process, cannot be taken rigidly adhere to today's technology and there should be the purpose of forward-thinking design to meet the technical requirements of the future development of the computer and allow the computer network design with high flexibility, compatibility and more space.

To Have the Maturity. On the basis of the advanced design, in conjunction with the existing technology has advanced and practical, choose a relatively mature computer technology, so that the computer network topology application has higher practicability.

Follow the Design Ideas of Entire Life Cycle. Computer network design, from the overall to be considered, the overall performance of the computer network designed to take the best performance of the system configuration to achieve optimal network performance.

\section{Factors Affecting the Reliability of the Computer Network}

The Impact of Computer Network Equipment on the Network Reliability. Effect of network devices on the network can be divided into user equipment reliability and transmission switching equipment in two ways. Mainly refers to the user equipment for the user terminal users, its network reliability is critical, because it is the key to determine whether the network reliability. We are running daily maintenance of computer networks and in fact, the main task is to ensure the absolute safety of the user terminal, if the ability to interact with the user terminal is higher, less reliable network security is also higher. In addition, the process of building a computer network, we will find that the computer network cabling system failures caused by the often cause the most headaches, because it is difficult to find the problem to staff inconvenience. Therefore, we should use standard network cabling systems and communication lines, in order to improve the safety and reliability of computer networks and to facilitate the future development of computer networks, in some important computer networks, and if in spite of the construction costs, then the wiring when should I use wire to facilitate the computer network if the line fails in the future, we can be timely switched. A hub is a single point of failure equipment, computer network hub will focus on a number of user terminals connected to it via the connected device spaced errors and other parts of the computer network, computer network defense constitute a guarantee of reliability, if a failure occurs hub, along with the user that it is connected also stopped working. Therefore, the computer 
network hub plays a great significance in improving the reliability of the computer network.

The Impact of Computer Network Management on the Network Reliability. Usually a large computer network is constituted by from different regions of the manufacturers of equipment and products, large-scale, structure and changeable. So we should use leading network management technology to ensure complete transmission of information, reduce the incidence of failure to reduce error in varying degrees, in order to improve the reliability of computer networks. Moreover, we should timely acquisition parameters of network operation, network operation statistical information to monitor the status of network operation in order to achieve a network error in time to find fault.

The Impact of Computers Network Topology on the Network Reliability. Computer network topology has a profound impact on the reliability of computer networks, we find that in practice, at different levels and in different areas of the scale network environments, often necessarily involves the participation of the network topology. Wherein the network topology is the main connection between the components of computer networks, as people continue to explore the computer network topology, and found a new concept and many network graph theory, such as restricted connectivity, restricted edge connectivity, limited by various concept Fault-tolerant diameter and the like. These parameters can be more accurate and provide a theoretical basis for the protection of computer network reliability.

\section{The Situation of Computer Network Reliability}

Computer Network Reliability refers to the time and conditions specified in the requirements, the computer network system is no exception, maintaining smooth network connection. That is, under certain conditions the function specified in the computer system, a computer network may be provided within the time normally required for operation of the network capacity. The reliability of computer networks is a key measure of a computer network system planning, design and operation. The topology of the computer network system uptime support plays an important role. Currently computer network has been a large number of applications in various sectors of society and it has become an integral part of people's life and production. In all areas of our country, we have a lot of making use of the computer network, which is also on the reliability of the network put forward a very high demand. Computer network system to undertake a large number of information storage, transmission, processing, involves a lot of personal information, business information, security information is the focus of attention. Necessity of development of things and as long as the two sides decided to confidential information, people would want to steal or destroy. For computer networks, some unscrupulous agencies in order to obtain personal information to seek illegitimate interests, to take a large number of illegal means to steal information and tampering of the more common methods are the use of network viruses or hacker techniques to take, which are on the computer network reliability It posed a severe challenge.

\section{Methods to Improve the Reliability of the Computer Network}

Use Fault-Tolerant Design. The computer network system according to line parallel lines designed by calculating the design allows the user terminal is connected at two points and so that you can solve the primary network redundancy, it can form a bi-directional network connection, improve network troubleshooting ability. This can effectively avoid the user can not use the network is faulty.

Dual Network Architecture Design. Redundant backup network needs to calculate the original network, improve fault tolerance of the entire network system. The solution is to use dual-network design, it is the principle of the computer network in the event of failure of the primary network, resulting in a network system is not working properly, the network backup system will replace the existing network system to ensure data transmission network to ensure safe operation of the computer network. Through the network fault-tolerant design, can protect the entire computer network security, the system is stable and reliable operation, improve the reliability of the network. 
Compared to fault-tolerant design solutions, the cost of the dual network design is relatively high, but the network design solutions ability to solve problems more thoroughly, make the system more stable, the safe operation of the entire network system security higher. Using this system, fault identification faster and easier to find the point of failure, troubleshooting is relatively simple. The fault-tolerant solutions lower cost, but compared to the double protection system on the network structure is slightly lower, also somewhat less ability to find fault for repair tools and techniques are relatively high.

Take the Overall Network System Design. With the progress of the times and social development, users of computer networks have become increasingly demanding, in order to respond to the needs of users, in the form of decentralized network structure of this function. This design can completely replace the centralized network architecture design is the need for the development of computer network technology. This design is the computer network of the extended internal structure to a certain extent, to effectively solve the problems in the operation, improve the reliability and security of the network.

Build a Hierarchy Layout Design. Computer network system consists of a network application layer, network services, network operating system, network layer and the physical hardware layer. Service layer is mainly used to provide network services, trying to databases, e-mail; the role of the application layer is mainly to meet the needs of network users; physical hardware layer network is the computer network hardware topology; network operating system is network layer software.

Consider the Life Cycle of the Whole Network. Improve the reliability of computer networks and it should also take into account the life cycle of the whole network, which is the need to consider the overall cycle cost of the system. In the management of the entire network system, it is necessary to establish a reasonable network systems, good planning and control. Under the protection of the network system performance without loss situation, taking cost-efficient way, the network system is to achieve the best state, to achieve the best price.

Strengthen the Network Management Monitoring. Improve the reliability of the computer network to strengthen the network management is essential and we must strengthen the overall network maintenance, reliable operation of the network in real-time monitoring and management. Since the development of network technology, a lot of large and complex computer networks are formed by different network devices made for such networks to avoid network failures and equipment downtime and other issues, therefore in order to avoid a system crash. This requires that in addition to the daily monitoring, but also professionals for maintenance treatment.

\section{Conclusion}

Looking at the future development of computer network reliability, security people on the network, the reliability requirements are getting higher and higher, both in daily life computer network, or in businesses working network environment, a computer network have been widespread attention and application, computer network reliability is the most important prerequisite to protect people in the online world tour, so the reliability of the computer network to improve security and optimize the network environment, it is imperative

\section{References}

[1] Jia Xinzhang, Li Jingyuan. Computer Engineering and Design, Vol. 6 (2014) No 53, p.25-26

[2] Wang Yunhui. Liaoning Institute of Science and Technology, Vol. 12 (2015) No 27, p.74-76

[3] Qian Xiyuan, Jing Jianfen, Hou XuSiem. Computer Engineering, Vol. 30 (2011) No 19, p.144-145

[4] Wang Kuailiang. Computer Knowledge and Technology, Vol. 29 (2012) No 27, p.21-23

[5] Sun Jing. Network Security Technology and Application, Vol. 8 (2013) No 27, p.57-60

江西省赣州市渡口路 7 号南阳东升 2 栋 2 单元 504 室 
手机号码: 13907078663 收件人: 赖敏 